\title{
Investigation of Optical Properties of Solochrome Dark Blue Dye Doped Polymer Films
}

\author{
Imad Al - Deen Hussein Ali Al - Saidi", Raghad Jabar \\ Department of Physics, College of Education for Pure Sciences, University of Basrah, Basrah, Iraq \\ *Corresponding author: al_saidi_imad@yahoo.com
}

\begin{abstract}
The optical properties of the pure poly(methylmethcrylate) (PMMA) polymer film and PMMA polymer films doped with Solochrome Dark Blue dye at different concentrations were investigated. The films were prepared using the casting technique. The optical absorption and transmission spectra of these films were recorded in the wavelength range 300 - $900 \mathrm{~nm}$ for different concentrations using UV-Vis double - beam spectrophotometer. The optical parameters of the Solochrome Dark Blue dye - doped polymer films, reflectance (R), absorption coefficient $(\alpha)$, extinction coefficient $(\kappa)$, refractive index $(\mathrm{n})$, optical and electrical conductivities $\left(\sigma_{\mathrm{opt}}\right.$ and $\left.\sigma_{\text {elect }}\right)$, and optical energy band gap $\left(\mathrm{E}_{\mathrm{g}}\right)$, were calculated. The effect of the dye concentration on these parameters was studied. The obtained results show that the Solochrome Dark Blue dye is a promising material for applications in photonic and optoelectronic devices.
\end{abstract}

Keywords: optical properties, optical parameters, Solochrome Dark Blue dye, dye - doped polymer films, optical energy band gap

Cite This Article: Imad Al - Deen Hussein Ali Al - Saidi, and Raghad Jabar, "Investigation of Optical Properties of Solochrome Dark Blue Dye Doped Polymer Films." Journal of Materials Physics and Chemistry, vol. 5, no. 1 (2017): 32-38. doi: 10.12691/jmpc-5-1-4.

\section{Introduction}

Nonlinear optical materials have received much interest because of their potential applications in optical devices such as, light emitting diodes, solar cells, optical sensors, optical signal processing, optical switching, optical power limiting, and optoelectronic devices [1-15]. The optical and electrical properties of wide range of materials, including organic and inorganic materials, semiconductors, and polymer materials, have been studied to develop practical optical devices [16-23]. Among these materials, organic dyes are of great interest owing to their potential application related to the nonlinear optical (NLO) properties. Organic dyes are currently attracting considerable attention and emerged as the most promising materials for devices applications owing to their high nonlinearity and ultra-fast responses, Polymers have also received significant interest due to their various applications in many scientific branches and materials technology [24,25,26,27,28]. There has been a large need for nonlinear optical materials that can be employed in optical devices applications. The polymeric materials can be modified when they doped with suitable organic dyes and their optical nonlinearities are considerably affected. This could lead to significant improvement in the optical and electrical properties of these materials. In the present study, we chose the Solochrome Dark Blue dye, which has excellent nonlinear optical properties, as a dopant nonlinear material and the poly(methylmethacrylate) (PMMA) as a host material because it is has several advantages of characteristics flexibility, ease of fabrication, good transparency, photo thermal stability, high damage threshold for the intense laser beams [3,29], as well as it has a relatively low cost.

There are various techniques which can be used to prepare the polymer films [30-35]. Among these techniques, casting is a good, simplest, and easy technique to prepare the polymer films. For our study, we were used this technique to prepare the pure poly(methylmethcrylate) (PMMA) polymer film and PMMA polymer films doped with Solochrome Dark Blue at different concentrations.

In the present paper, the optical properties of the prepared polymer films were investigated and their optical parameters, reflectance $(\mathrm{R})$, absorption coefficient $(\alpha)$, extinction coefficient $(\kappa)$, refractive index (n), optical and electrical conductivities $\left(\sigma_{\text {opt }}\right.$ and $\left.\sigma_{\text {elect }}\right)$, and optical energy band gap $\left(\mathrm{E}_{\mathrm{g}}\right)$, were calculated. The effect of dye concentration on the values of these parameters was also studied.

\section{Theoretical}

The linear absorption coefficient $(\alpha)$ of an optical medium is usually described by the Lembert-Beer law as [36]

$$
\mathrm{I}=\mathrm{I}_{0} \mathrm{e}^{-\alpha \mathrm{L}}
$$

where $\mathrm{I}_{0}$ is the incident light intensity, $\mathrm{I}$ is the transmitted light intensity, and $\mathrm{L}$ is the length of the absorbing medium. If the absorbance (A) of the medium sample is defined by $\mathrm{A}=\mathrm{I}_{0} / \mathrm{I}$, then Eq. (1) can be written as follows [36,37]: 


$$
\mathrm{A}=\mathrm{e}^{\alpha \mathrm{L}}
$$

or it takes the form:

$$
\alpha=2.303 \frac{\mathrm{A}}{\mathrm{L}} .
$$

The extinction coefficient $(\kappa)$ of the medium sample is directly proportional to the absorption coefficient $(\alpha)$ according to the following relation [38]:

$$
\kappa=\frac{\alpha \lambda}{4 \pi}
$$

where $\lambda$ is the wavelength of incident light.

The refractive index (n) is one of the fundamental parameters of a material due to the close relation between $\mathrm{n}$ and the properties of material. It is given by the following relation [39]:

$$
\mathrm{n}=\left[\frac{1+\mathrm{R}}{1-\mathrm{R}}\right]+\left[\frac{4 \mathrm{R}}{(1-\mathrm{R})^{2}}-\kappa^{2}\right]^{1 / 2}
$$

where the optical reflectance $(\mathrm{R})$ of the material is given by the relation $[30,38,39]$ :

$$
\mathrm{R}=1-\left(\mathrm{Te}^{\alpha \mathrm{L}}\right)^{1 / 2}
$$

The optical conductivity of the material is given by the following relation [38]:

$$
\sigma_{\mathrm{opt}}=\frac{\alpha \mathrm{nc}}{4 \pi}
$$

where $\mathrm{c}$ is the velocity of light.

The electrical conductivity $\left(\sigma_{\text {elect }}\right)$ of the material is related to its optical conductivity $\left(\sigma_{\text {opt }}\right)$ according to the following relation [38]:

$$
\sigma_{\text {elect }}=\frac{2 \lambda \sigma_{\mathrm{opt}}}{\alpha} .
$$

The optical energy gap $\left(E_{g}\right)$ between the valence band and the conduction band of the material is an important quantity that characterize material. It is related to the absorption coefficient $(\alpha)$ and the incident photon energy $(\mathrm{h} v)$ according to the following relation $[36,39,40]$ :

$$
(\alpha \mathrm{h} v)=\mathrm{C}\left(\mathrm{h} v-\mathrm{E}_{\mathrm{g}}\right)^{\mathrm{m}}
$$

where $\mathrm{h}$ is the Planck's constant, $v$ is the frequency of incident photons, $\mathrm{C}$ is a constant, its value depends on the transition probability, and $\mathrm{m}$ is an index, its value depends on the type of the electronic transition [40]. In the present work, the analysis of UV-Vis. absorption spectra indicates that the dye - doped polymer films have allowed indirect transitions, therefore $m=2$ was used in Eq. (9) for the determination of the value of $\mathrm{E}_{\mathrm{g}}$.

\section{Materials and Experimental Details}

The Solochrome Dark Blue dye has molecular formula

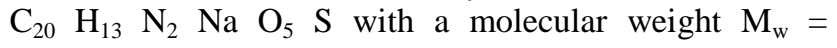
$416.39 \mathrm{~g} /$ mole.. The chemical structure of this dye is shown in Figure 1. Poly(methylmethacrylate) (PMMA) polymer in the form of small grains (crystalline polymer) with high purity and good optical transparency was used in this study. Samples of pure PMMA polymer film and dye - doped polymer films were prepared by using the casting method. A certain weight of Solochrome Dark Blue dye was dissolved in a mixed solvent of Tetra Hydro Furan (THF) with little quantity of methanol, suitable solvent for both the dye and the polymer. Then required weight of host PMMA polymer was added. The mixture was stirred at room temperature using a magnetic stirrer until a homogeneous solution was formed. This solution was diluted by the solvent and samples of solutions with different dye concentrations were obtained. Then, proper quantities of the prepared solutions were cast on thin glass slides and kept for drying for 48 hours at room temperature. Dye - doped polymer film samples with different concentrations $(0.05,0.06,0.07,0.08,0.09 \mathrm{mM})$ ) and an average thickness of $0.9 \mathrm{~mm}$, were obtained. The obtained polymer films were examined and found that they are uniform with good optical quality.

The Ultraviolet - Visible (UV-Vis) absorption spectra of prepared pure PMMA polymer film and Solochrome Dark Blue dye - doped polymer films were characterized by using Cecil double-beam spectrophotometer (model CE-75000) with the wavelength range 190 - $1100 \mathrm{~nm}$. An uncoated glass slide was used as a reference sample for the absorption measurement.

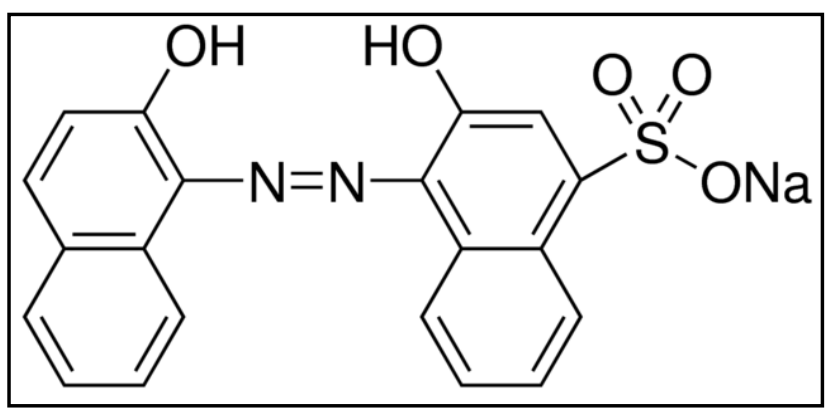

Figure 1. Chemical structure of Solochrome Dark Blue dye

\section{Results and Discussion}

The absorbance (A), the transmittance (T), and the reflectance $(\mathrm{R})$ spectra of the Solochrome Dark Blue dye doped polymer films at different dye concentrations were recorded over the wavelength range $300-900 \mathrm{~nm}$ using the UV-Vis. spectrophotometer and are shown in Figure 2, Figure 3, and Figure 4, respectively. In Figure 2, the absorbance spectra of dye - doped polymer films exhibited strong broad band with peak absorption around $530 \mathrm{~nm}$. The absorbance spectra, in Figure 2, give absorbance values of $10.0 \%-17.4 \%$ for concentration range 0.05 $\mathrm{mM}-0.09 \mathrm{mM}$. The transmittance of the Solochrome Dark Blue dye - doped polymer films reached to about $79 \%$ at the low dye concentration $0.05 \mathrm{mM}$ and it is decreased with increased the dye concentration, as shown in Figure 3. It is seen from Figure 4 that the spectrum curves of the reflectance of the Solochrome Dark Blue dye - doped polymer films show behaviors similar to those of the absorbance; this is attributed to the correlation between reflectance and the absorbance. The value of $\mathrm{R}$ at the low dye concentration $0.05 \mathrm{mM}$ is only $6.4 \%$ in the 
visible wavelengths region and increases with increasing the dye concentration. This value reached to $9.2 \%$ at the dye concentration $0.09 \mathrm{mM}$; at wavelength $\lambda \approx 530 \mathrm{~nm}$.

The values of the absorption coefficient $(\alpha)$ of the Solochrome Dark Blue dye - doped polymer films were calculated using Eq. (3). The variation of $\alpha$ with the photon energy $(\mathrm{h} v)$ at different dye concentrations is displayed in Figure 5. It is seen that the value of $\alpha$ increases with increasing the photon energy (hv) until reached to its maximum value at incident photon energy $\mathrm{h} v \approx 2.5 \mathrm{eV}$ and then starts to decrease with increasing hv. This is clearly evident with high dye concentrations. It is also seen that increasing dye concentration leads to significant increase in the value of $\alpha$. Over the dye concentration range $(0.05-0.09) \mathrm{mM}$, the value of $\alpha$ varies within the range $(2.3-4.0) \mathrm{cm}^{-1}$.

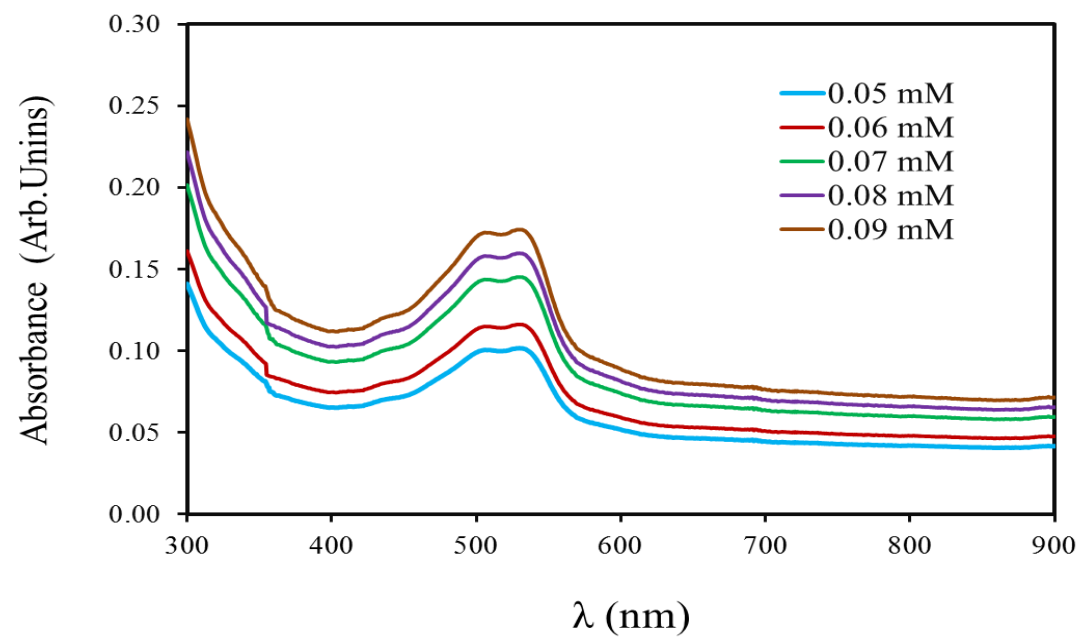

Figure 2. UV - Vis linear absorption spectra of the Solochrome Dark Blue dye - doped polymer films at different dye concentrations

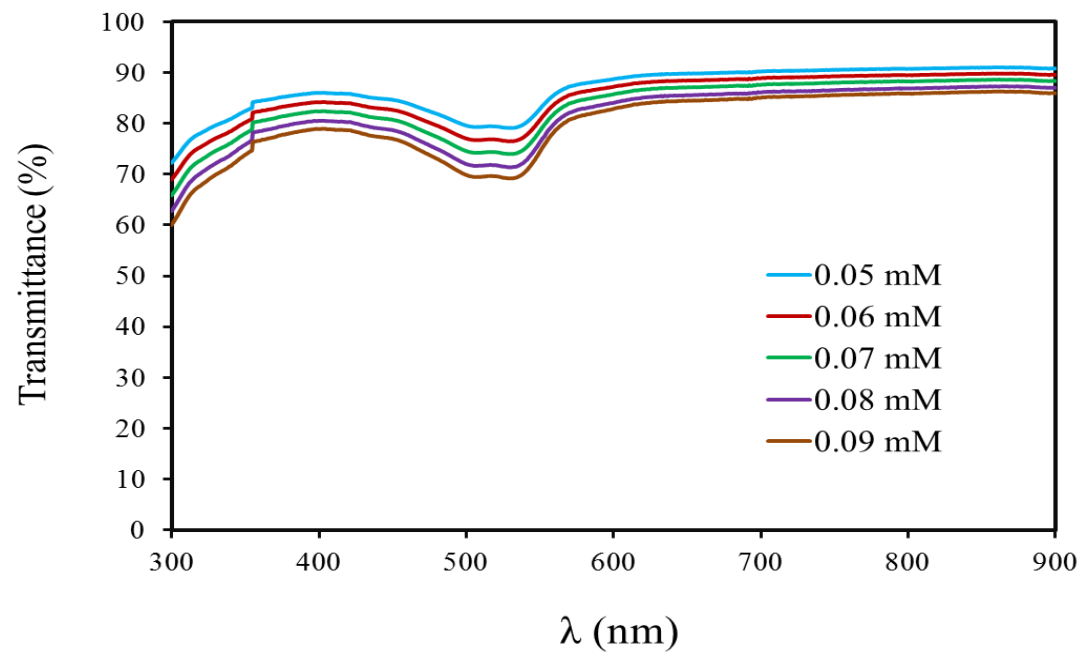

Figure 3. UV-Vis transmittance spectra of the Solochrome Dark Blue dye - doped polymer films at different dye concentrations

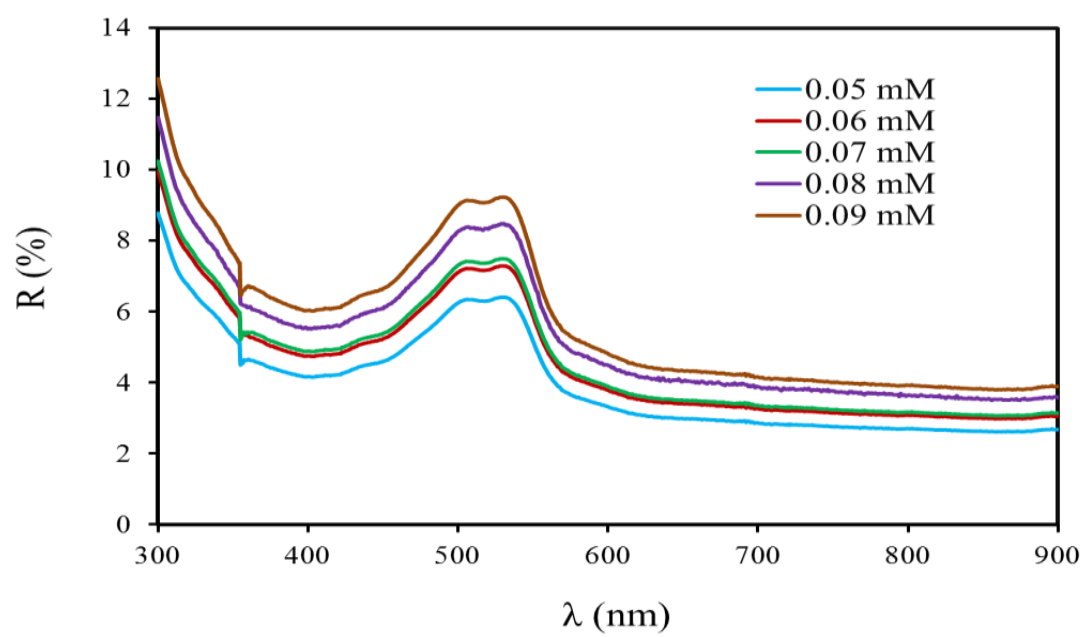

Figure 4. UV-Vis reflectance (R) spectra of the Solochrome Dark Blue dye - doped polymer films at different dye concentrations 


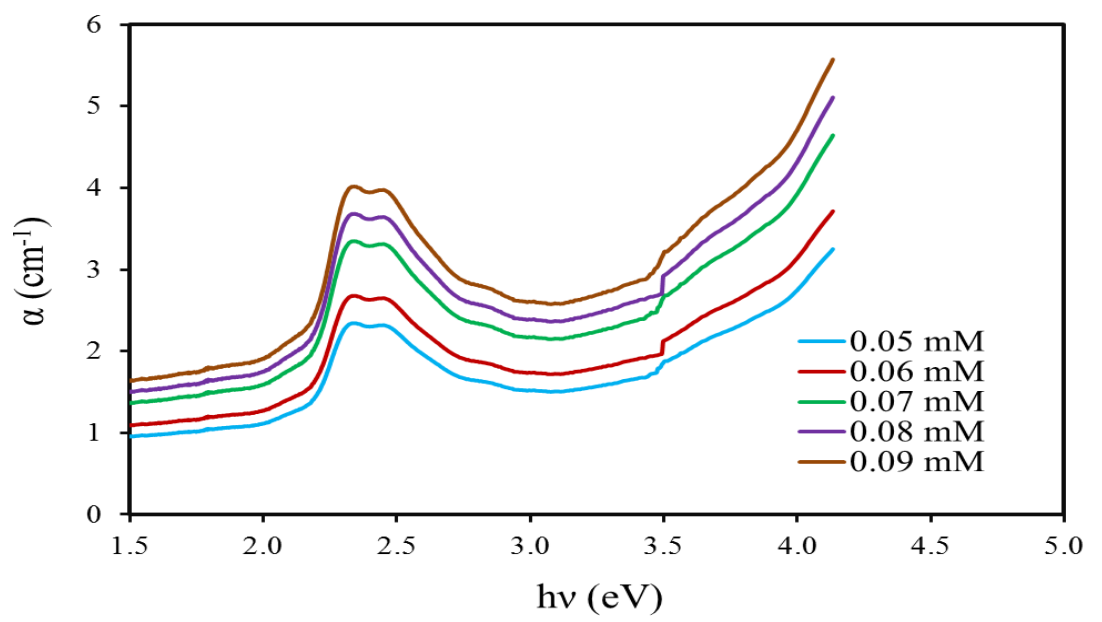

Figure 5. The absorption coefficient $(\alpha)$ of the Solochrome Dark Blue dye - doped polymer film versus the incident photon energy (hv), for different dye concentrations

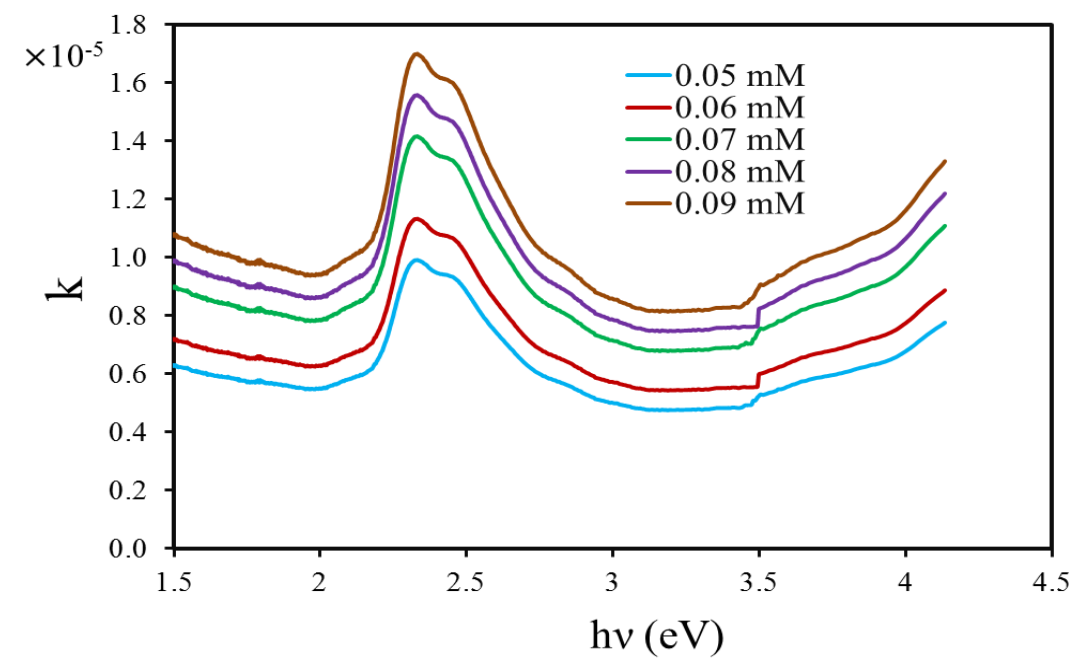

Figure 6. The extinction coefficient $(\kappa)$ of the Solochrome Dark Blue dye -doped polymer film versus the incident photon energy (hv), for different dye concentrations

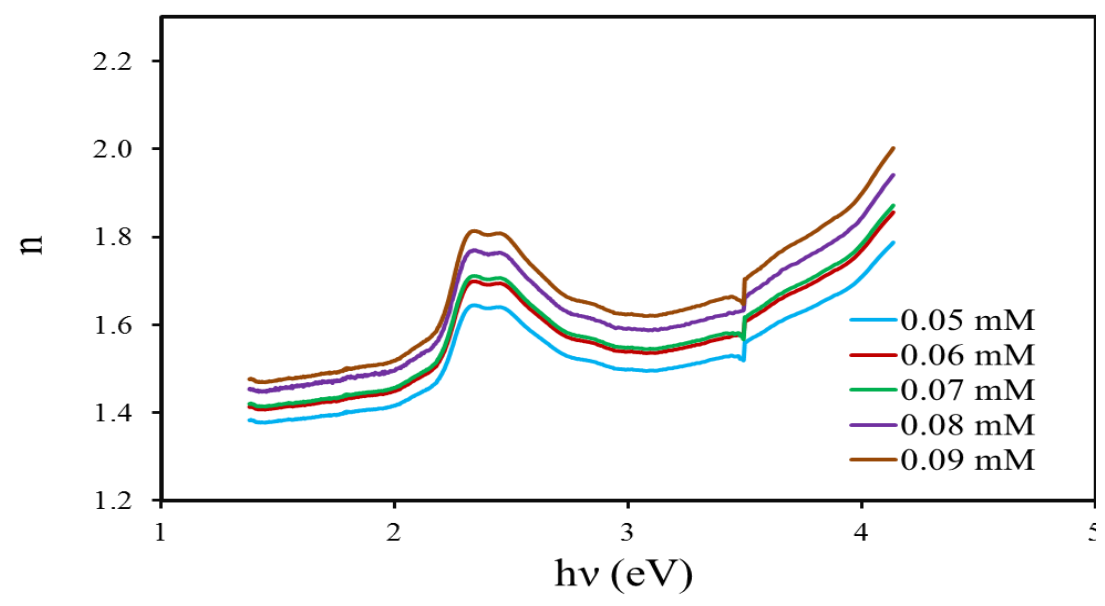

Figure 7. The refractive index (n) of the Solochrome Dark Blue dye - doped polymer film versus the incident photon energy (hv), for different dye concentrations

Using the values of the corresponding $\alpha$ and Eq. (4), the values of the extinction coefficient $(\mathrm{k})$ for the Solochrome Dark Blue dye - doped polymer films at different dye concentrations were calculated for different values of incident photon energy $(\mathrm{hv})$. The variation of $\mathrm{k}$ of the Solochrome Dark Blue dye - doped polymer film with the photon energy (hv) is plotted in Figure 6 for different dye concentrations. It is noticed that the variation of $\mathrm{k}$ with $\mathrm{h} v$ has behavior similar to that of $\alpha$ because of the direct relation between the two constants through the relation (5). The value of $\mathrm{k}$ varies within the range $(1.0-1.7) \times 10^{-5}$ for the dye concentration range $(0.05-0.09) \mathrm{mM}$. 
Figure 7 shows the variation of the refractive index (n) of the Solochrome Dark Blue dye - doped polymer film with incident photon energy $(\mathrm{h} v)$ for different dye concentrations. The value of $\mathrm{n}$ is within the range (1.6 - 1.8) for the concentration range $(0.05-0.09) \mathrm{mM}$. It is obviously that $\mathrm{n}$ of the dye - doped polymer film depends on the photon energy and its value reached to maximum at $h v \approx 2.3 \mathrm{eV}$.

We used, respectively, Eqs. (7) and (8) to calculate $\sigma_{\text {opt }}$ and $\sigma_{\text {elect }}$ of the Solochrome Dark Blue dye - doped polymer films. The variations of $\sigma_{\text {opt }}$ and $\sigma_{\text {elect }}$ with incident photon energy are shown in Figure 8 and Figure 9, respectively, for different dye concentrations. The evaluated value of $\sigma_{\text {opt }}$ is within the range $(0.3-0.6) \times 10^{10}$ $(\mathrm{sec})^{-1}$, while the value of $\sigma_{\text {elect }}$ is within the range $(1.4$ 1.6) $\times 10^{5}\left(\mathrm{~S} . \mathrm{cm}^{-1}\right)$, over the dye concentration range $(0.05-0.09) \mathrm{mM}$. It is seen that the value of $\sigma_{\text {elect }}$ is high at the low photon energies and decreases with increasing the incident photon energy (hv).

The values of the optical energy band gap $\left(E_{g}\right)$ for the Solochrome Dark Blue dye - doped polymer films were estimated from the intersect of the extrapolated linear part of the plot of $(\alpha h v){ }^{1 / 2}$ versus the incident photon energy (h $v$ ) with abscissa, as shown in Figure 10. The obtained values of $E_{g}$ for the Solochrome Dark Blue dye - doped polymer films are given in Table 1 . It is seen that the value of the optical energy band gap of pure PMMA polymer sample (undoped polymer) is equal to $4.73 \mathrm{eV}$, and reduced with increased the concentration of the Solochrome Dark Blue dye in the PMAA polymer (from $4.73 \mathrm{eV}$ to $1.83 \mathrm{eV}$ at dye concentration $0.09 \mathrm{mM}$ ), this is attributed to the increase in absorption coefficient $(\alpha)$ as a result of introducing dopant atoms and hence the value of $\mathrm{E}_{\mathrm{g}}$ will be decreased. From Table 1, one can deduce that, in the presence of dye, the optical energy band gap of the pure PMMA polymer film is modified significantly and its value decreased by increasing the dye concentration. This is probably due to the creation of point defects within the optical energy band gap.

Table 1. The obtained values of optical energy band gap $\left(E_{g}\right)$ for the pure PMMA polymer film and the Solochrome Dark Blue dye doped polymer films at different dye concentrations

\begin{tabular}{|c|c|}
\hline Sample & $\begin{array}{c}\text { Optical energy band gap }\left(\mathrm{E}_{\mathrm{g}}\right) \\
(\mathrm{eV})\end{array}$ \\
\hline PMMA & 4.73 \\
\hline SDB $0.05 \mathrm{mM}$ & 1.92 \\
\hline SDB $0.06 \mathrm{mM}$ & 1.90 \\
\hline SDB $0.07 \mathrm{mM}$ & 1.88 \\
\hline SDB $0.08 \mathrm{mM}$ & 1.86 \\
\hline SDB $0.09 \mathrm{mM}$ & 1.83 \\
\hline
\end{tabular}

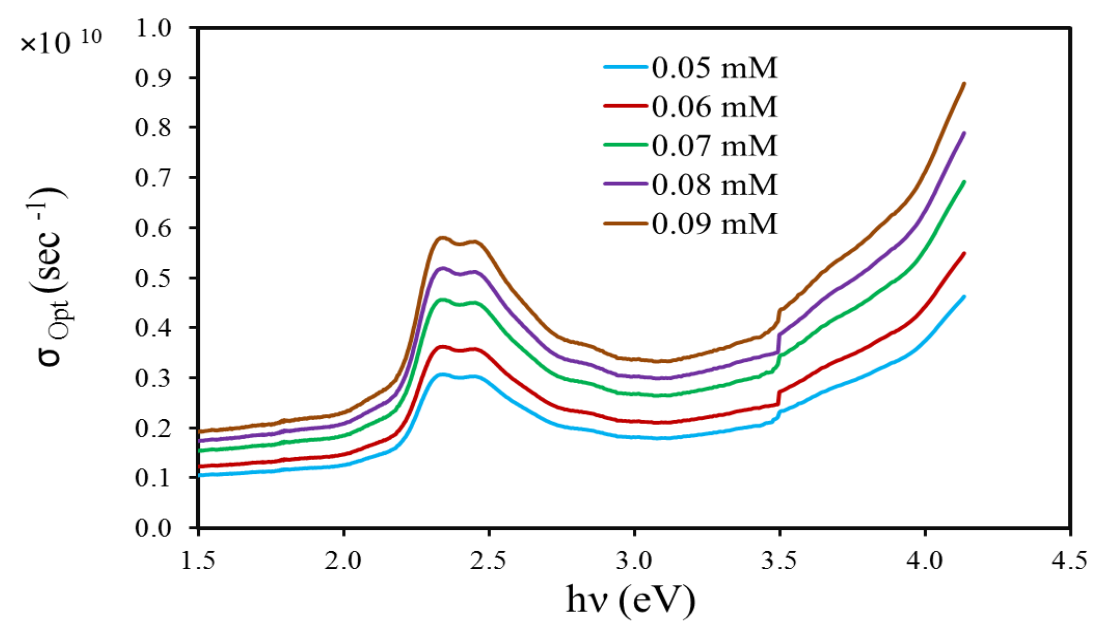

Figure 8. The variation of the optical conductivity $\left(\sigma_{\mathrm{opt}}\right)$ with the incident Photon energy (hv) for the Solochrome Dark Blue dye - doped polymer film at different dye concentrations

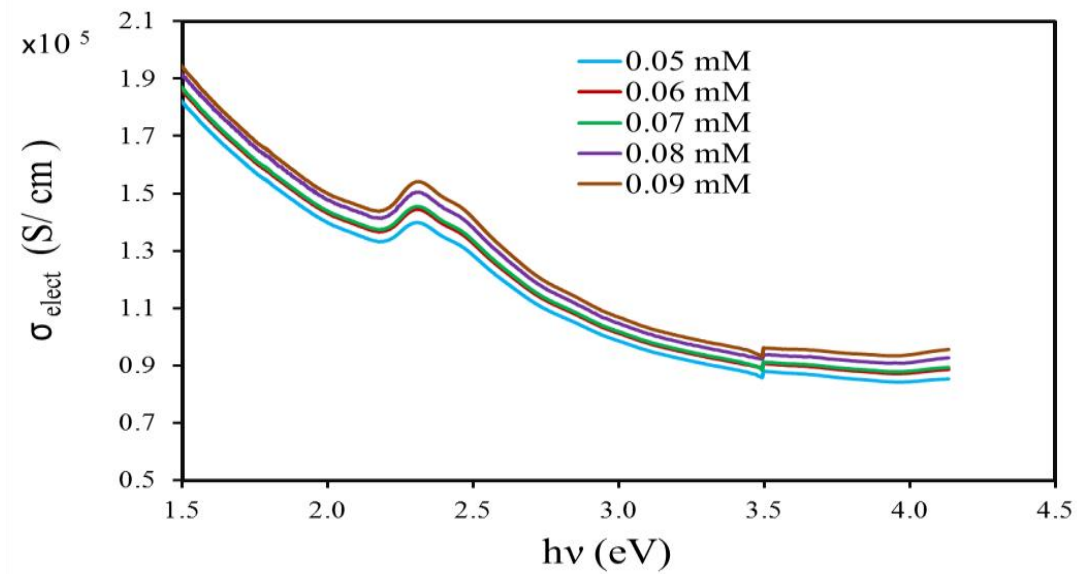

Figure 9. The variation of the electrical conductivity $\left(\sigma_{\text {elect }}\right)$ with the incident photon energy $(h v)$ for the Solochrome Dark Blue dye - doped polymer film at different dye concentrations 

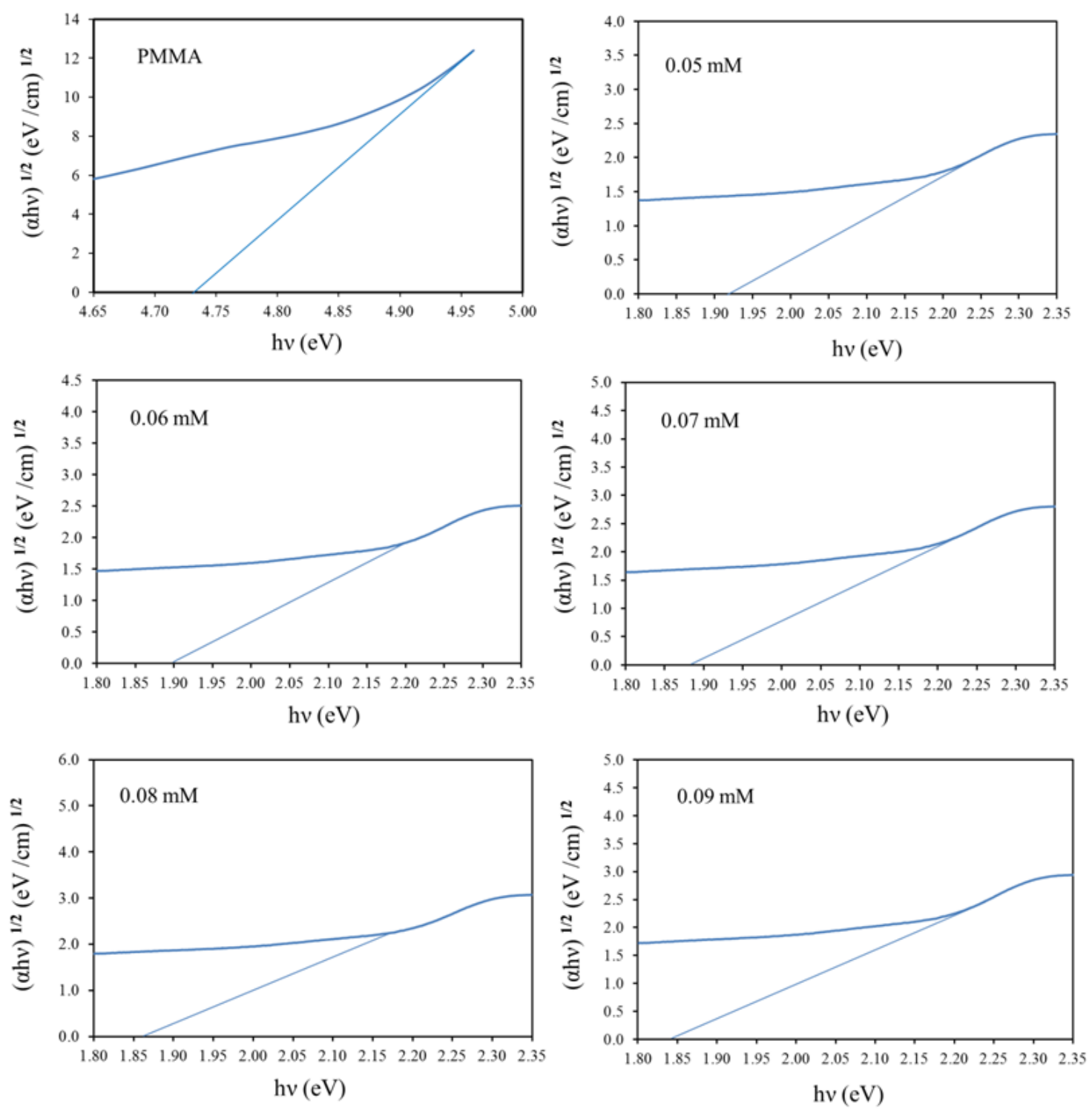

Figure 10. The plots of $(\alpha h v)^{1 / 2}$ versus hv for Pure PMMA polymer film and Solochrome Dark Blue dye - doped polymer films at different dye concentrations

\section{Conclusions}

The pure and Solochrome Dark Blue dye - doped polymer films were prepared using the casting technique and their optical properties were investigated for different dye concentrations. The optical parameters of the dye doped polymer films were determined for different dye concentrations. Results showed that the optical properties of the PMMA polymer film were appreciably modified in the presence of the organic Solochrome Dark Blue dye. The optical energy band gap $\left(\mathrm{E}_{\mathrm{g}}\right)$ value of the pure PMMA polymer shows significant decreasing with an increasing concentration of Solochrome Dark Blue dye. The obtained results from the investigation suggest that the prepared Solochrome Dark Blue dye - doped polymer films are suitable for photonic device applications.

\section{References}

[1] Kim, D. Y., Cho, H. N. and Kim, C. Y., "Blue Light Emitting Polymers", Progress in Polymer Science, 25 (2000), 1089-1139.
[2] S. C. Lo and P. L. Burn, "Development of Dendrimers: Macromolecules for Use in Organic Light-Emitting Diode and Solar Cells", Chemical. Reviews, 107 (2007), 1097-1116.

[3] S.-L Yeh, C.-Y. Zhu, and S.-W. Kuo, "Transparent Heat Resistant PMMA Copolymers for Pacing Light-Emitting Diode Materials", Polymers, 7 (2015), 1379-1388.

[4] W. Wu, et. al., "Efficient and Stable Dyesensitized Solar Cells Based on Phenothiazine Sensitizers with Thiophene Units", J. Mater. Chem., 20 (2010), 1772-1779.

[5] S. Qu, W. Wu, J. Hua, C. Kong, Y. Long, and H. Tian, "New Diketopyrrolopyrrole (DPP) Dyes for Efficient Dye-Sensitized Solar Cells", J. Phys. Chem. C, 114 (2010), 1343-1349.

[6] K. D. Seo, et. al., "Coumarin Dyes Containing Low-Band-Gap Chromophores for Dye-Sensitised Solar Cells", Dyes and Pigments, 90 (2011) 304-310.

[7] M. Fitra, I. Daut, N. Gomesh, M. Irwanto, and Y.M. Irwan, "Dye Solar Cell using Syzigium Oleina Organic Dye", Energy Procedia, 36 (2013), 341-348.

[8] A. Sacco, et. al., "Dye-Sensitized Solar Cell for a Solar Concentrator System", Solar Energy, 125 (2016), 307-313.

[9] G. Harsanyi, Polymer Films in Sensor Applications: Technology, Materials, Devices and their Applications (Technomic Publishing Company Inc., Pennsylvania, USA, 1995).

[10] J. M. Hales, S. Barlow, H. Kim, S. Mukhopadhyay, J. - L. Bredas, J. W. Perry, and S. R. Marder, "Design of Organic Chromophores for All - Optical Signal Processing Applications", Chem. Mater., 26 (2014), 549-560. 
[11] F. Z. Henari and S. Cassidy, Nonlinear Optical Properties and All - Optical Switching of Congo Red in Solution, Optik, 123 (2013), 711-714.

[12] I. Al-D. H. Al-Saidi, and S. Al-D. Abdulkareem, "Nonlinear Optical Properties and Optical Power Limiting of Leishman Dye Using Z- Scan Technique. J. of Mater. Sci.: Mater. Electron., 20 (2015), 2713-2718.

[13] Imad Al - Deen Hussein A. Al - Saidi and Saif Al - Deen Abdulkareem, "Study of Nonlinear Optical Properties and Optical Power Limiting of Leishman Dye Using Z-Scan Technique", Indian J. Phys., 89 (2015), 1199-1203.

[14] Imad Al - Deen Hussein A. Al - Saidi and Saif Al - Deen Abdulkareem, "Nonlinear Optical Properties and Optical Power Limiting Effect of Giemsa Dye Polymer Films", Opt. Laser Technol., 82 (2016), 150-156.

[15] Y. Guo, C. K. Kao, E. H. Li, and K.S. Chang, Nonlinear Photonics: Nonlinearities in Optics, Optoelectronics and Fiber Communications (The Chinese University Press and SpringerVerlag, Berlin, Germany, 2002).

[16] J. Zyss, (Ed.), Molecular Nonlinear Optics: Materials, Physics, Devices (Academic Press, INC., New York, USA, 1994).

[17] H. S. Nalwa, (Ed.), Handbook of Advanced Electronic and Photonic Materials and Devices (Academic Press, New York, 2001).

[18] M. Cada, "Nonlinear optical devices", Opt. Pur. y Apl. 38 (2005), $1-10$.

[19] O. Ostroverkhova, (Ed.), Handbook of Organic Materials for Optical and Opto-electronic Devices: Properties and Applications (Woodhead Publishing Ltd., UK, 2013).

[20] T. Kobayashi, (Ed.), Nonlinear Optics of Organics and Semiconductors, Springer Proceedings in Physics, Vol. 36, (Springer-Verlag, Berlin, Germany, 1989).

[21] W. Nie, "Optical Nonlinearity: Phenomena, Applications, and Materials", Adv. Mater., 5 (1993), 520-545.

[22] P. Günter, (Ed.), Nonlinear Optical Effects and Materials (Springer-Verlag, Berlin, Germany, 2000).

[23] H. Zollinger, Color Chemistry: Synthesis, Properties and Applications of Organic Dyes and Pigments, 3rd ed, (Wiley-VCH, Cambridge, 2003).

[24] H. G. Elias, An Introduction to Polymer Science, (Weinheim, NewYork, USA, 1997).
[25] N. G. C. McCrum, P. C. Buckley, and B. Bucknall, Principles of Polymer Engineering, 2nd ed., (Oxford University Press, New York, USA, 1997).

[26] K. -S. Lee, (Ed.), Advances in Polymer Science, Vol. 158, (Springer-Verlag, Berlin / Heidelberg, Germany, 2002).

[27] L. H. Sperling, Introduction to Physical Polymer Science, (John Wiley \&Sons, Inc., Hoboken, 2006).

[28] C. Fleischmann, M. Lievenbrück, and H. Ritter, "Polymers and Dyes: Developments and Applications", Polymers, 7 (2015), 717-746.

[29] A. Costela, et. al., "Laser Performance of Pyrromethene 567 Dye in Solid Polymeric Matrices with Different Cross-Linking Degrees", Journal of Applied Physics, 90, (2001), 3159-3166.

[30] I. Eckertova, Physics of Thin Films, 2nd ed., (Plenum Press, NewYork, USA, 1986)

[31] H. Frey and H. R. Khan, (Eds.), Handbook of Thin Film Technology (Springer-Verlag, Berlin, Germany, 2015).

[32] K. S. Kim, H. W. Kim, and N. H. Kim, "Structural Characterization of $\mathrm{ZnO}$ Film grown on $\mathrm{SiO}$ by the RF Magnetron Sputtering", Physica B. 334 (2003), 343-346.

[33] F. S. Chien, C. R. Wang, Y. L. Chain, and R. J. Wu, "Fast Response Ozone Sensors with $\mathrm{ZnO}$ Nanorods Grown by Chemical Vapor Deposition", Sens. Actuators B: Chemical, 144 (2010). 120-125.

[34] B. L. Zhu, X. Z. Zhao, F. H. Su, and X. G. Wu, "Low Temperature Annealing Effect on the Structure and Optical Properties of ZnO Fllm Grown by Pulsed Laser Deposition", Vacuum, 84 (2010). 1280-1286.

[35] Z. R. Khan, M. S. Khan, M. Zulfequar, and M. S. Khan, "Optical and Structural Proper-2 Ties of $\mathrm{ZnO}$ thin Films Fabricated by Sol Gel Method, Mater. Sci. Appl., 2 (2011). 340 -345.

[36] N. F. Mott, and A. E. Davis, Electronic Process in Non-Crystalline Materials, 2nd ed., (University Press, Oxford, UK, 1979).

[37] D. E. Gray, (Ed.), American Institute of Physics Handbook, 3rd ed., (McGrow Hill Book Co., New York, USA, 1982).

[38] J. I. Pankove, Optical Processes in Semiconductors (Prentice Hall, New York, USA, 1971).

[39] T. S. Moss, Optical Properties of Semiconductors (Academic Press, New York, USA, 1974).

[40] J. Tauc, Amorphous and Liquid Semiconductors, Vol. 159 (Plenum Press, New York, USA, 1974). 